\title{
L'efficacia del diritto nelle Vorstudien di Theodor Geiger, spunti e riflessioni
}

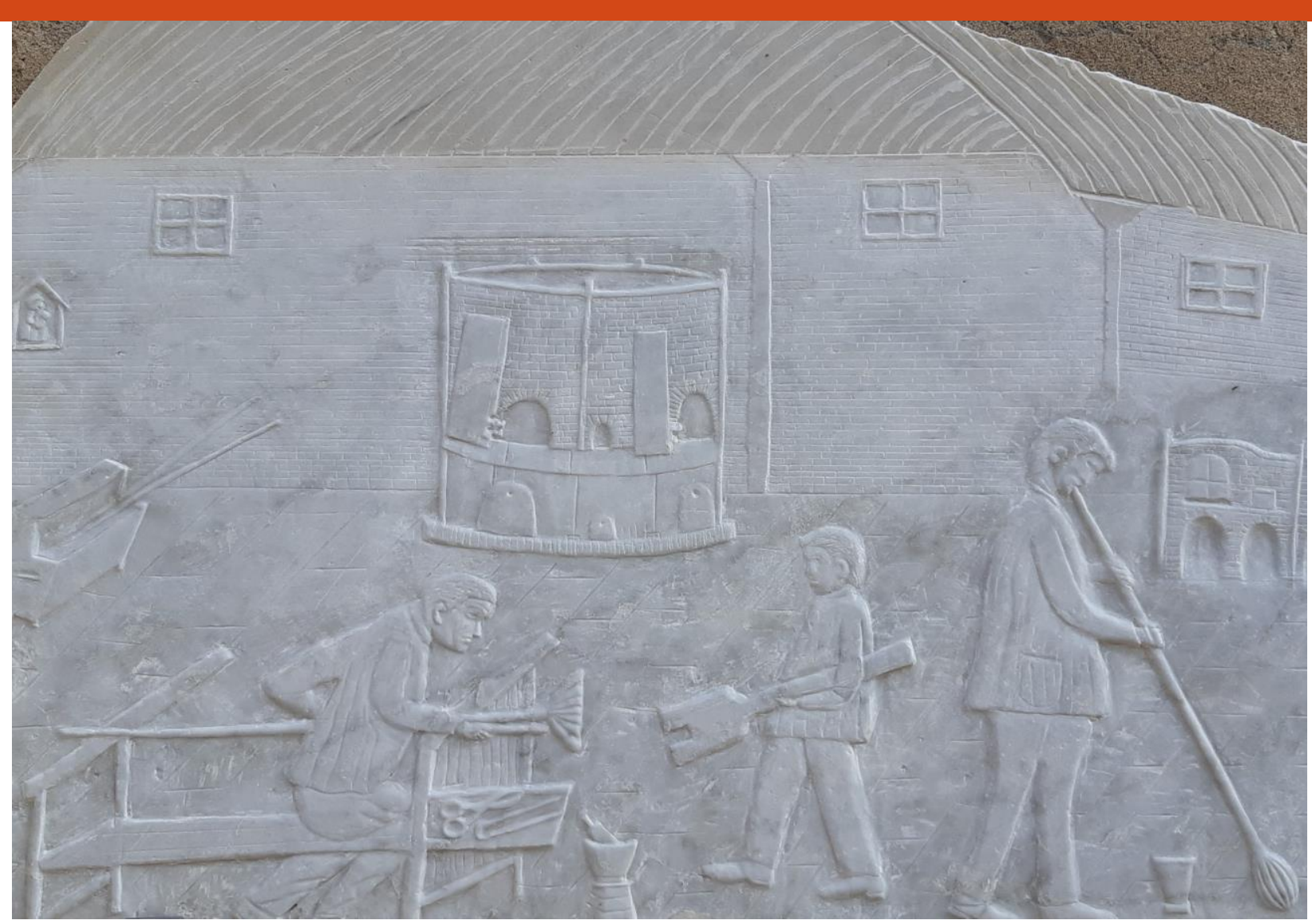

2021 ANNO VI NUMERO 12

di Enrico Damiani di Vergada Franzetti DOI https://doi.org/10.54103/2531$6710 / 17354$ 


\section{L'EFFICACIA DEL DIRITTO NELLE VORSTUDIEN DI THEODOR GEIGER, SPUNTI E RIFLESSIONI}

\section{di Enrico Damiani di Vergada Franzetti}

\section{THE EFFECTIVENESS OF THE LAW IN THE VORSTUDIEN OF THEODOR GEIGER}

\section{Riassunto}

Il presente lavoro analizza il tema dell'efficacia (inefficacia) del diritto alla luce delle riflessioni svolte sull'argomento da Theodor Geiger nel volume dal titolo Vorstudien zu einer Soziologie des Rechts (1947), al fine di individuare le variabili che appaiono maggiormente in grado di influenzarla.

Parole chiave: Diritto; Efficacia; Theodor Geiger

\section{Abstract}

This paper analyses the issue of the effectiveness (ineffectiveness) of law in the light of Theodor Geiger's reflections on the subject in his book Vorstudien zu einer Soziologie des Rechts (1947), in order to identify the variables that seem most likely to influence it.

Keywords: Law; Effectiveness; Theodor Geiger

Autore: Enrico Damiani di Vergada Franzetti, Dottore di ricerca in Sociologia del diritto, Università degli studi di Milano, e cultore della materia in Sociologia del diritto, Sociologia del lavoro, Sociologia della Devianza.

Articolo soggetto a revisione tra pari a doppio cieco

Articolo ricevuto il 02.2.20 approvato il 01/02/21 


\section{Efficacia del diritto, sistema semiotico e interpretazione delle norme}

L'applicazione e la disapplicazione del diritto non rappresentano soltanto il tema fondamentale della sociologia del diritto, ma debbono anche costituire oggetto di un attento studio teorico-empirico poiché l'ipotesi di un diritto che viene costantemente e senza eccezione alcuna osservato, sembra persino meno interessante per il sociologo del diritto di quella della sua disapplicazione (Bettini 1988: 33 ss.; Ferrari 1997: 261; Febbrajo 1979; 1984; 2009: 83-84; 2018)'.

L'efficacia normativa dipende da una moltitudine di fattori riconducibili a variabili specifiche, dipendenti e indipendenti, concernenti l'azione sociale e comunicativa (Geiger [1947]2018: 262; Febbrajo 2009: 94), che vanno descritte e analizzate, comprese e spiegate con l'ausilio della ricerca teorico-empirica.

Il presente lavoro intende non solo ricostruire, sia pure in termini incompleti e sommari, il quadro concettuale dell'efficacia del diritto alla luce delle riflessioni svolte sul tema da Geiger, ma anche analizzare i temi e i problemi ad essa connessi da un punto di vista semiotico comunicativo.

La semiotica giuridica, settore specialistico della semiotica generale, studia il diritto come insieme di segni: il sistema giuridico, rete di interazioni comunicative dipendenti dagli attori sociali, può essere definito come un circolo semiotico attraversato da messaggi di varia natura che pongono innumerevoli problemi interpretativi e definitori (Conte 1962; 1986; 1995; 2011; Scarpelli 1969; 1985; Tarello 1975; 1980; Jori 1995: 305; Kalinowski 1993: 547; Ferrari 1997: 80).

Le norme, messaggi comunicativi che attraversano lo spazio discorsivo strutturato da una relazione multiadica tra emittenti e riceventi, non sempre individuati e reciprocamente noti, costituiscono il mezzo di una comunicazione normativa multilaterale che coinvolge innumerevoli soggetti e media. Gli attori sociali che partecipano a questo processo riflettono la cultura, il sistema di valori dell'ambiente cui appartengono, cui si rivolgono e rendono conto: ogni attore interagisce con gli altri, riflette le diverse percezioni, i vincoli psichici e sociali in funzione dei quali agisce (Ferrari 1997: 163; Petrażycki 1909-1910). I messaggi, le norme che i soggetti comunicano e si scambiano, esprimono aspettative e richieste comportamentali soggette a numerosi processi interpretativi, almeno tanti quanti sono i partecipanti alla comunicazione se non di più,

\footnotetext{
${ }^{1}$ L'articolo è frutto della rielaborazione del paper presentato al Convegno 22-23 maggio 2018, Sala Napoleonica, Palazzo Greppi, Via S. Antonio 12, Milano, L'eredità di Theodor Geiger per le scienze giuridiche.
} 
suscitando reazioni diverse che vanno dall'adesione al rifiuto, da atteggiamenti cognitivi a quelli normativi (Galtung 1960: 213 ss.): aderire alle aspettative significa accettare il modello normativo e l'interpretazione proposti, sottrarvisi o rifiutarle significa proporre o contrapporre interpretazioni, modelli normativi alternativi e diversi su cui incidono altrettante variabili. In questa prospettiva se è vero che il processo comunicativo dei messaggi normativi rende difficile l'individuazione della fonte primaria dell'atto comunicativo perché chi comunica è stato previamente informato in una catena potenzialmente senza fine, mentre persino chi crea un messaggio riflette ciò che esisteva prima dell'invenzione, vero è allora che i messaggi, le norme, nelliterazione comunicativa tra i soggetti che ascoltano, interpretano e comunicano, mutano in modo incessante il loro significato (originario) (Ferrari 1997: 163-4; Geiger [1947]2018: 283-98). In questo senso l'interpretazione dei messaggi giuridici rappresenta un problema sia per il giurista pratico che per il sociologo del diritto che studia la comunicazione sociale attraverso il diritto e sul diritto: se è vero, afferma Geiger, che la comunicazione giuridica è facilitata dalla chiusura autoreferenziale dei sistemi giuridici basati su di una semiotica giuridica e una semantica convenzionale costituita da meta-regole che stabiliscono i modelli da seguire nella formulazione, nell'applicazione e nell'interpretazione delle norme (Geiger [1947]2018: 305), vero è tuttavia, sottolinea ancora l'autore, che il diritto, le norme espresse con segni linguistici, possono essere interpretati unicamente ricorrendo ad altri segni linguistici, con l'ovvio corollario che ogni atto interpretativo che voglia precisare il significato di una norma può allontanarsi sia dal significato originario, sia da quello espresso nell'atto interpretativo che lo precede moltiplicando in tal modo i giudizi di efficacia o inefficacia di una norma, almeno tante volte quante sono le interpretazioni possibili ovvero i soggetti coinvolti (Geiger [1947]1964a: 58 ss.; [1947]2018: 282-302; Ferrari 1997: 80; Febbrajo 2009: 1003). In questi termini l'attività interpretativa riguardante le parole svolta mediante l'uso di parole, nell'ambito di un processo semiotico illimitato, comporta che ogni messaggio normativo, recepito e trasmesso, venga re-interpretato cambiando di contenuto nel corso di ogni passaggio successivo: se, rileva Geiger, l'attività comunicativo-interpretativa vertente sugli enunciati normativi proclamativi, il cui significato è prescritto come vincolante, è sempre creativa, modificativa e idonea a porre nel nulla il significato originario di una proposizione normativa, di ogni precedente atto di interpretazione (Geiger [1947]2018: 290, 293; Hart 1965: 146 ss.; Kelsen 1967: 117 ss.; Tarello 1990), allora medesime caratteristiche contraddistinguono l'efficacia 
giuridica (Geiger [1947]1964a: 58 ss.; Geiger [1947]2018: 282-302; Ferrari 1997: 167; Febbrajo 2009: 100103).

Si tratta di aspetti che possono essere meglio compresi allorché, osserva Geiger, si consideri come gli enunciati normativi proclamativi, espressioni linguistiche indirizzate a qualificare atti e accadimenti concreti, presentano sempre inevitabili margini di indeterminatezza semantica (Geiger [1947]1964a: 58 ss.; [1947]2018: 298; Kelsen 1967: 68 ss., 98; Ferrari 1997: 217; Febbrajo 2009: 100-3) risultando variamente interpretabili. Ogni interprete, non solo il legislatore che produce la legge in conformità alla costituzione, ma anche il giudice che emette la sentenza in conformità della legge, le parti contrattuali che agiscono in base alle disposizioni di un negozio giuridico, in generale i ruoli sociali connessi all'azione giuridica non sono mai compiutamente vincolati sul piano logico dall'enunciato normativo che interpretano (Geiger [1947]1964a: 58 ss.; [1947]2018: 290, 296; Kelsen 1967: 117 ss.; [1979]1985: 378 ss.; Ferrari 1997: 217; Febbrajo 2009: 100-3).

Se, afferma Geiger, una norma è un modello ideale caratterizzato da vaghezza semantica cui rapportare un'evidenza reale, in grado di assumere significati diversi in relazione alle diverse interpretazioni possibili, espressione dell'incidenza di altrettante variabili, allora non vi è dubbio che il giudizio di efficacia si caratterizzi per un elevato grado di contingenza e relatività, spesso dissimulata dagli operatori del diritto (Geiger [1947]2018: 292-293). Le norme giuridiche, rileva l'autore, per l'ambiguità e l'incertezza semantica che le caratterizza (Geiger [1947]2018: 298), intenzionale o meno che sia, producono molteplici effetti in relazione ai soggetti che, secondo differenti progetti d'azione, con modalità diverse e non sempre dichiarati scopi e interessi, vengono coinvolti dal processo di formulazione, applicazione e interpretazione del diritto. La consapevolezza del carattere relativo e contingente del concetto di efficacia giuridica, se non conduce ad allontanarlo dal quadro teorico dell'indagine sociologico-giuridica, in quanto si eliminerebbe una categoria concettuale necessaria per la comprensione-spiegazione dell'agire sociale, allora deve indurre i sociologi del diritto a concentrarsi sulle, ad analizzare le, variabili che appaiono maggiormente in grado di condizionarla attraverso lo svolgimento di ricerche teorico-empiriche mirate. Nei paragrafi che seguiranno si descriveranno, alla luce delle riflessioni svolte sul tema da Geiger, alcune delle variabili che appaiono in grado di incidere sull'efficacia giuridica sotto il profilo comunicativo concernente il rapporto tra norme (messaggi) e azioni (comunicative). 


\section{La costruzione sociale dell'efficacia giuridica: la formulazione, l'applicazione e}

\section{l'interpretazione del diritto}

Se l'efficacia del diritto nella visione realistica geigeriana esprime l'idea che l'aderenza e lo scostamento delle azioni rispetto alle norme, l'applicazione di una sanzione, siano aspetti empiricamente osservabili e quantificabili (Geiger [1947]1964a; [1947]1964b; [1947]2018: 255-276; Febbrajo 2009: 89-95; Ferrari 1997: 261), allora è opportuno da subito sottolineare, come ha rilevato l'autore, che sia la qualificazione di un comportamento come conforme o deviante rispetto ad una norma, nei termini di ottemperanza e trasgressione, sia l'applicazione di una sanzione da parte di soggetti istituzionalizzati a ciò deputati, tendano a variare in funzione dei significati socialmente attribuiti o attribuibili alle norme, per l'operare di variabili che influenzano i processi sociali e comunicativi riguardanti la formazione, l'interpretazione e l'applicazione del diritto (Febbrajo 2009: 100-3; Geiger [1947]2018: 283-91). Un aspetto che risulta tanto più evidente se si considerano le norme da un punto di vista comunicativo, come fatti comunicativi o atti di comunicazione, e in tal modo verranno considerate nello svolgimento del presente lavoro. Che una norma sia o meno efficace, che un'azione trasgredisca o meno un enunciato normativo proclamativo, che si debba o meno comminare una sanzione, costituisce, come ha chiaramente osservato Geiger, il risultato di un'attività comunicativo-interpretativa di carattere definitorio svolta da alcuni soggetti nell'ambito di processi più o meno istituzionalizzati (Geiger [1947]1964: 149 ss.; [1947]2018: 283-91; Febbrajo 2009: 95-99). L'attività definitoria svolta, la qualificazione proposta non consistono in una connotazione naturale del modello normativo o del concreto comportamento considerati, semmai esprimono un - il - punto di vista (soggettivo) da cui un soggetto guarda la realtà (Petrażycki 1909-1910; Fittipaldi: 2012; 2016), costituiscono il mezzo, il fine attraverso cui si realizza la costruzione sociale dell'efficacia giuridica (Ortega y Gasset 19461947: 197-203; Geiger [1947]1964a: 169 ss.; [1947]2018: 293; Berger \& Luckmann [1966]1969; Treves 19871988: 333; Febbrajo 2009: 98; Damiani di Vergada Franzetti 2018: 154-64). Sotto questo profilo occorre rilevare come il procedimento definitorio, qualificativo concernente il giudizio di efficacia può sia precedere che seguire l'azione oggetto di valutazione, potendo persino consistere nell'emanazione di una nuova norma, ovvero nella diversa interpretazione di una norma praticata ed esistente, o, ancora e paradossalmente come ha rilevato Geiger, nella reintroduzione nell'ordinamento giuridico del principio di retroattività della legge 
(Marx [1842]1975: 177 ss.; Geiger [1947]1964a; [1947]2018: 283-92, 302; Febbrajo 2009: 100-3), tema ripreso e approfondito da Morris Ghezzi (2017: 55-62; 2018: 21-66).

Non vi è dubbio, osserva l'autore, che le menzionate operazioni definitorie e qualificative determinino non solo il passaggio di molte norme dal campo dell'efficacia a quello dell'inefficacia e viceversa, ma anche lo spostamento di una moltitudine di comportamenti sociali dal campo dell'ottemperanza a quello della trasgressione e viceversa, con comminatoria o meno di una sanzione: la diversa collocazione è frutto di opzioni comunicativo-interpretative assunte dagli attori sociali, dai ruoli sociali connessi all'azione giuridica alla luce delle variabili in grado di condizionare i processi comunicativo-interpretativi concernenti la formazione, l'interpretazione e l'applicazione delle norme (Geiger [1947]2018: 283-92; Ferrari 1997: 180). Sotto questo profilo la dimensione di relatività e di contingenza dell'efficacia del diritto se esclude il carattere assoluto dell'alternativa secca tra le opposte rappresentazioni dell'efficacia e dell'inefficacia giuridica, dell'ottemperanza e della trasgressione cui segue o meno la comminatoria di una sanzione, dunque della formulazione, dellinterpretazione e applicazione di una norma in un senso piuttosto che in un altro, allora evidenzia un continuum di posizioni intermedie tra gli opposti estremi concettuali, laddove si collocano non solo tutte le infinite possibili e impensabili azioni umane, ma anche e paradossalmente, se così possiamo definirle, tutte le relative "efficacie giuridiche" (Geiger [1947]2018: 294-98). Non esiste infatti l'efficacia o l'inefficacia del diritto in termini assoluti, come non esiste l'ottemperanza o la trasgressione di una norma in termini assoluti, e neppure esiste un solo modo per infliggere una sanzione, e nemmeno esiste un solo modo di produrre, interpretare e applicare le norme; semmai esiste un'ampia zona di spazio inesplorata, al cui interno si collocano non solo tutte le possibili e impensabili azioni umane, degli attori sociali, dei ruoli sociali connessi all'azione giuridica in rapporto alle proposizioni normative, ma anche le innumerevoli variabili che sono in grado, come in effetti accade, di influenzarle (Geiger [1947]1964a: 113; Van de Kerchove \& Ost 1995; Ferrari 1997: 180; Febbrajo 2009: 102).

Se il giudizio di efficacia giuridica viene colto in relazione all'elevato grado di contingenza e variabilità che caratterizza l'interazione comunicativa concernente la formulazione, l'interpretazione e l'applicazione delle norme (Febbrajo 2009: 101-3), allora le conclusioni cui si giunge non consistono necessariamente nella sfiducia in ordine alla possibilità di svolgere un'analisi concettuale vertente sul rapporto fra norme e azioni, perché semmai è vero proprio il contrario: il carattere di relatività e di contingenza del concetto di efficacia 
e inefficacia del diritto, del giudizio di ottemperanza o di trasgressione sulle norme cui segue o meno l'applicazione di una sanzione, del processo di formazione, interpretazione e applicazione del diritto, del rapporto tra norme e azioni, deve motivare i sociologi del diritto nell'impegnarsi a studiare, a indagare le diverse variabili, interne ed esterne al sistema giuridico, economiche, culturali, burocratiche, politiche, militari, sociali, ecc., che influenzano in termini probabilistici (Febbrajo 2009: 103), non solo i processi comunicativi vertenti sul rapporto tra norme e azioni, ma anche gli attori e i ruoli sociali connessi all'azione giuridica (Ghezzi 1995; Ferrari 1997: 180), in definitiva l'efficacia giuridica.

\section{Le variabili in grado di incidere sull'efficacia giuridica da un punto di vista comunicativo}

I sistemi giuridici tendono a differenziarsi in base al livello più o meno elevato di efficacia che li caratterizza, intesa come maggior o minor corrispondenza tra il quadro ideale rappresentato dal diritto e la realtà concreta: tra i compiti della sociologia del diritto vi è appunto quello di individuare mediante lo svolgimento di ricerche teorico-empiriche, le variabili che maggiormente influiscono su tale distanza (Treves 1987-88: 187 ss.). In questa prospettiva occorre rilevare come l'efficacia giuridica dipenda da una moltitudine di fattori riconducibili a specifiche variabili, dipendenti e indipendenti, in grado di incidere sia sulle forme di mediazione simbolica intese come messaggi (il diritto), che sull'azione sociale comunicativa posta in essere dagli attori sociali (azione giuridica), variabili che vanno osservate, descritte e analizzate ricorrendo alla ricerca teorico-empirica: senza pretesa di completezza ed esaustività, sulla scorta delle riflessioni svolte da Geiger, descriviamone alcune vertenti sui messaggi normativi e sull'azione giuridica comunicativa.

L'incertezza semantica, osserva Geiger ([1947]2018: 298), incide sull'efficacia del diritto poiché aumenta il livello di contingenza che connota i processi comunicativi concernenti le norme. Il lessico giuridico dovrebbe tendere alla massima chiarezza per garantire una comunicazione efficace: nonostante il linguaggio giuridico sia denominato, classificato, ordinato dalla dogmatica giuridica, secondo criteri semantici ed ermeneutici ritenuti vincolanti dagli specialisti del diritto, esso incorre negli effetti distorsivi propri della comunicazione giuridica (Ferrari 1997: 100). Si tratta di una variabile che può incidere sul significato di una norma ampliandone l'efficacia in senso sociologico-giuridico sotto il profilo degli effetti prodotti: se tali effetti sono tanti quante le possibili interpretazioni, allora dipendono dal grado di incertezza semantica di ciascuna norma. 
L'utilizzo di differenti lessici incide sull'efficacia della norma in quanto la variabilità di significato delle parole, nonostante l'esistenza di scambi tra i diversi linguaggi, aumenta il livello di contingenza dei processi comunicativi riguardanti le norme (Ferrari 1997: 166). Inoltre il linguaggio giuridico vertendo su di un'estesa gamma di oggetti, eventi e azioni umane, spesso ricorre, nel precisare il significato delle espressioni utilizzate, sia a sistemi semiotici (linguaggi) diversi da quello giuridico, sia a significati convenzionali suoi propri, ingenerando variabilità terminologica anche in quei settori, primariamente in quello giuridico, in cui dovrebbe vigere il dogma della certezza (ibid.). Si tratta di variabili che possono influire sul significato di una norma ampliandone l'efficacia in senso sociologico-giuridico ovvero sotto il profilo degli effetti prodotti, che, se sono tanti quante sono le interpretazioni possibili, allora dipendono dai diversi lessici, dai sistemi semiotici cui si è fatto ricorso o dai significati convenzionali eventualmente adottati: si tratta di variabili che Geiger ha rilevato rientrando nel tema dell'incertezza semantica delle norme (Geiger [1947]2018: 298).

Il contesto discorsivo in cui vengono utilizzati i segni giuridici, variandone il significato, incide sull'efficacia del diritto, rendendo difficoltosa la comunicazione giuridica (Morris 1938; Ferrari 1997: 80): un aspetto analizzato dalla semiotica "pragmatica" settore specialistico della semantica giuridica. Si tratta di una variabile che, influendo sul significato di una norma, può relativizzare il concetto di efficacia in senso sociologico giuridico sotto il profilo degli effetti realizzati, che se risultano essere tanti quanti sono le possibili interpretazioni di una proposizione normativa, allora dipendono dai diversi contesti in cui le espressioni giuridiche vengono utilizzate: una variabile che Geiger sembra aver analizzato, rientrando nel tema dell'incertezza semantica delle norme.

Il trascorrere del tempo, osserva Geiger ([1947]2018: 296-97, 309, 312), incide sull'efficacia del diritto, modificando il senso socialmente condiviso delle parole: non solo espressioni giuridiche tratte dal linguaggio comune semanticamente vaghe, ma anche concetti fondamentali ed espressioni di elevato di tecnicismo, tendono a mutare di significato con il decorso del tempo (Ferrari 1997: 165). Si tratta di una variabile che, condizionando il significato di una norma, può ampliarne l'efficacia in senso sociologico-giuridico sotto il profilo degli effetti realizzati, che se risultano essere tanti quanti sono le interpretazioni possibili, allora dipendono dai diversi significati socialmente condivisi prodotti dal decorso del tempo.

Lo spazio fisico discorsivo, sostiene Geiger ([1947]2018: 311, 312), incide sull'efficacia del diritto sia perché la medesima espressione può assumere un significato diverso da luogo a luogo, sia perché la trasposizione 
spaziale dei messaggi da una comunità linguistica a un'altra ne muta il significato risultando molte parole del lessico giuridico di un paese non traducibili in quello di un altro e viceversa (Candian, Gambaro \& Pozzo 1992; Ferrari 1997: 165). Si tratta di una variabile che può incidere sul significato di una proposizione normativa variandone l'efficacia in senso sociologico-giuridico sotto il profilo della molteplicità degli effetti prodotti, che se risultano essere tanti quanti sono le possibili interpretazioni, allora dipendono dai diversi spazi discorsivi entro cui i messaggi giuridici circolano: una variabile che Geiger sembra aver considerato rientrando nella trattazione generale del tema della vaghezza semantica delle norme.

Lo spazio comunicativo entro cui le norme giuridiche circolano, sottolinea Geiger ([1947]2018: 294-5, 310, 311, 315), influisce sull'efficacia del diritto poiché la cultura, l'ideologia professionale, l'opinione pubblica, le finalità con cui tale spazio recepisce, filtra e trasforma i messaggi giuridici che riceve, mutano il significato delle norme. Il discorso giuridico vertente sulle norme si distingue e assume significati diversi a seconda dei "gruppi semiotici" destinatari dei messaggi: la tipologia dell'uditorio e i relativi interessi influiscono sulla natura e sul significato delle norme (Jackson 1985: 284 ss.; Ferrari 1997: 166-7). Si tratta di una variabile che può influire sul significato di una norma relativizzandone l'efficacia sotto il profilo degli effetti realizzabili, i quali se risultano essere tanti quanti sono le possibili interpretazioni, allora sono in funzione dei destinatari dei messaggi normativi.

Le diverse culture giuridiche degli utenti del discorso normativo, afferma Geiger ([1947]2018: 294-5, 311), incidono sull'efficacia del diritto rendendo difficoltosa la comunicazione giuridica (Ferrari 1997: 80; Febbrajo 2009: 73; D’Alessandro 2018). Si tratta di una variabile che, operando sul significato di una proposizione normativa, può influire sull'efficacia in senso sociologico-giuridico sotto il profilo degli effetti prodotti, che, se risultano tanti quante sono le possibili interpretazioni, allora dipendono dalle diverse culture giuridiche degli utenti del discorso normativo.

La società contemporanea si caratterizza per una sovraproduzione normativa che, sostiene Geiger ([1947]2018: 306-8), incide sull'efficacia del diritto rendendo persino impossibile non solo conoscere il significato delle norme, ma addirittura ottemperarvi. L'oscurità, la contraddittorietà, la lacunosità delle disposizioni normative, aggiungendosi alle continue modifiche e integrazioni legislative, dottrinali e giurisprudenziali, rendono incomprensibili le norme spesso sollevando questioni che sembravano risolte, ingenerando continua confusione e incertezza (Bosello 1990; Ferrari 1997: 267); contrasti normativi 
insanabili che, se inducono gli attori sociali a trasgredire le norme nel momento stesso in cui ne adempiono altre, allora rendono impossibili i comportamenti in esse previsti imponendo requisiti assurdi, condizioni impossibili che impediscono persino il compimento delle attività richieste (Sgubbi 1990; Ferrari 1997: 268). Si tratta di una variabile che può incidere profondamente sul significato di una norma, ampliandone l'efficacia in senso sociologico-giuridico dal punto di vista degli effetti prodotti: si tratta di effetti che, se risultano diversi quante sono le possibili interpretazioni di una norma, allora sono in funzione della sovraproduzione legislativa, dottrinale e giurisprudenziale.

La conoscenza e l'opinione che hanno i destinatari delle norme, rileva Geiger ([1947]2018: 316), incide sull'efficacia del diritto trattandosi di aspetti ad essa strettamente correlati (Treves 1987-1988: 284-8; Cotterrell 1992: 138 ss.), ma secondo nessi di relazione che, se risultano parziali, allora si caratterizzano per modalità niente affatto scontate (Ferrari 1997: 264; Febbrajo 2009: 111). Se è vero che non si può ottemperare volontariamente una norma che non si conosce, vero è che l'osservanza di una proposizione normativa può dipendere dall'imitazione, dall'adesione spontanea ai valori ad essa sottesi (Ferrari 1997: 2645). Inoltre se è vero che una norma non condivisa ma conosciuta induce alla trasgressione, è altrettanto vero che si può ottemperare una norma non condivisa per inerzia, per timore della sanzione, per rispetto del sistema normativo ritenuto legittimo, illegittimo, finanche ingiusto: la tendenza sociale al rispetto di una norma non condivisa sul piano etico-politico è spesso imputabile all'assenza di alternative d'azione (Podgòrecki 1991; Ferrari 1997: 265). Si tratta di variabili che possono influire sul significato di una norma relativizzandone l'efficacia in senso sociologico-giuridico sotto il profilo degli effetti prodotti, che se risultano essere tanti quanti sono le interpretazioni svolte dai destinatari di una disposizione normativa, allora variano al variare dell'opinione (pubblica) e della conoscenza che essi hanno del diritto.

La mancata conoscenza del significato socialmente condiviso delle espressioni giuridiche utilizzate, sostiene Geiger ([1947]2018: 316), l'ignoranza del codice linguistico adottato, con riguardo al senso letterale delle parole, incide sull'efficacia del diritto per la confusione che produce nei destinatari delle norme circa il significato attribuito o attribuibile alle espressioni giuridiche (Ferrari 1997: 164). Si tratta di un aspetto che, se raramente coinvolge gli specialisti del diritto, più spesso riguarda i gestori della comunicazione sociale impegnati in un'attività comunicativa senza conoscere adeguatamente il codice della comunicazione che utilizzano o a cui si riferiscono. Si tratta anche in questo caso di una variabile che può influire sul significato 
di una norma relativizzando il concetto di efficacia in senso sociologico-giuridico sotto il profilo degli effetti prodotti, che se sono tanti quante le possibili interpretazioni del modello normativo, allora variano in funzione dei significati alternativi proposti dai soggetti che ignorano quelli socialmente condivisi: una variabile che Geiger sembra aver analizzato rientrando nel più ampio tema dell'opinione e conoscenza delle norme.

L'implementazione delle proposizioni normative, osserva Geiger ([1947]2018: 301-2), intendendo riferirsi con tale espressione alle risorse materiali e simboliche, alle attività materiali, intellettuali e organizzative poste al servizio delle norme, incide sull'efficacia del diritto, assicurando la realizzazione di strutture e infrastrutture necessarie per lo svolgimento delle attività connesse al diritto (Bardach 1977; Mayntz 1980; Kagan 1982; Blankenburg 1986: 59 ss.; 1988: 291-3; 1995: 115; Podgòrecki 1991: 195 ss.; Podgòrecki, Alexander \& Shields 1996; Ferrari 1997: 265). Le norme spesso non raggiungono gli obbiettivi prefissati poiché non sono supportate da adeguati mezzi implementativi (Paliero 1990: 438-43), quantitativi di tipo economico e qualitativi di tipo organizzativo: la disorganizzazione burocratica spesso ostacola le attività connesse alle norme dotate di copertura finanziaria, mentre l'assenza di copertura economica sovente impedisce le attività correlate alle norme nonostante queste risultino burocraticamente ben organizzate (Bettini 1983; 1990; Ferrari 1997: 266). Si tratta di una variabile che può influire sull'operatività di una norma relativizzando il concetto di efficacia in senso sociologico-giuridico sotto il profilo degli effetti prodotti, che variano in funzione delle risorse stanziate al servizio delle norme.

La sanzione negativa, rileva Geiger ([1947]2018: 262), influenza l'efficacia del diritto condizionando l'attitudine degli attori sociali a ottemperare o a trasgredire una norma, sebbene ciò non accada secondo modalità scontate o nessi di relazione usuali (Febbrajo 2009: 121; Petrażycki 1909-1910; Fittipaldi 2012; 2016). La pena capitale ad esempio non costituisce sempre il mezzo di contrasto più efficace per la commissione dei reati più gravi, perché la comparazione dei dati relativi a paesi che la prevedono con quelli che la escludono, se non elimina l'incidenza di ulteriori variabili, allora evidenzia una ridotta efficacia come mezzo di prevenzione sociale (Ferrari 1997: 267). Sotto questo profilo occorre poi aggiungere che il decorso del tempo riduce l'efficacia della pena di morte perché, se è vero che la reclusione costituisce un'effettiva minaccia per chi non l'ha mai subita, vero è che la minaccia di più gravi sanzioni come l'ergastolo o la pena capitale intimorisce assai meno il reo che le ha già sperimentate (Friedman [1975]1978; Ferrari 1997: 267). 
Si tratta di una variabile che può influire sull'operatività delle norme relativizzando il concetto di efficacia in senso sociologico-giuridico sotto il profilo degli effetti prodotti, che variano in funzione delle motivazioni e degli schemi percettivi propri dei destinatari del diritto.

In ultima istanza l'adozione di una definizione di diritto in termini sociologico-giuridici oppure dogmaticogiuridici, sottolinea Geiger ([1947]2018: 277-83), incide sull'efficacia del diritto non solo sotto il profilo della qualificazione in termini giuridici di un fenomeno sociale, ma anche e soprattutto sotto il profilo della sua azionabilità in termini processuali mediante il ricorso ad istituzioni specializzate (Geiger [1947]2018: 264-5). Si tratta di un aspetto che può incidere sulla efficacia giuridica in termini sociologico giuridici dal punto di vista degli effetti prodotti, che, intesi sotto il profilo della realizzabilità processuale del diritto, tendono ad esistere o meno al variare della definizione di diritto adottata.

Come Geiger ha avuto modo di evidenziare, in un caso si tratta di variabili riguardanti le norme, come l'incertezza semantica, l'utilizzo di lessici differenti, il ricorso a sistemi semiotici alternativi, la definizione di significati convenzionali, il contesto discorsivo, il trascorrere del tempo, lo spazio fisico discorsivo. Variabili che, se non eliminano la rilevanza del discorso giuridico "interno" ed "esterno", cui i giuristi credono di rapportarsi, allora evidenziano come la vincolatività di tali significati, dei criteri di significazione adottati sia più apparente che reale; come il lavoro del giurista, la comunicazione giuridica, siano più improntati all'aleatorietà che non alla certezza; come i principi ermeneutici esprimano più verosimilmente un discorso retorico che non logico (Nelken \& Febbrajo 1993: 87 ss.; Ferrari 1997: 100). Nell'altro si verte invece su variabili riguardanti i soggetti, come lo spazio comunicativo (gruppi semiotici), le diverse culture giuridiche, la sovraproduzione normativa, la conoscenza e l'opinione sulle norme, la mancata conoscenza del significato socialmente condiviso delle espressioni giuridiche utilizzate, l'implementazione delle norme, la sanzione negativa, l'adozione di una definizione di diritto. Variabili che se non eliminano la possibilità di fondare una previsione sull'azione giuridica e sui ruoli sociali connessi (giuristi, legislatori, burocrati, giudici, gli avvocati), allora evidenziano non solo che si tratta di previsioni da assumere in termini probabilistici, ma anche che sulla relativa affidabilità e sui loro nessi di relazione, pesano in modo determinante alcune variabili esterne al sistema giuridico: variabili tipiche di altri sistemi d'azione, come quello politico ed economico, in grado di produrre importanti conseguenze non solo sul rapporto tra norme e azione, sulla nozione di efficacia giuridica (Ferrari 1997: 260), ma anche sulle funzioni manifeste o latenti degli enunciati normativi. 
Sotto quest'ultimo profilo vi sono proposizioni normative che pur risultando in vigore in quanto formalmente emanate, tuttavia non vengono applicate: si tratta di simboli che è utile mostrare in pubblico, utilizzare nell'arena politica, spendere nei rapporti sociali e giuridici. Se l'apparenza della legge, evocando l'immagine della giustizia, costituisce l'effetto "simbolico" delle norme giuridiche, allora "la funzione simbolica" del diritto consiste nel persuadere e nell'orientare i consociati, nel legittimare il potere, nel giustificare la distribuzione dell'autorità mediante l'esibizione di simboli normativi. Si tratta delle cosiddette "leggi manifesto" che immesse nel circolo mediatico, sebbene prive di effetti concreti perché inapplicate, ma formalmente in vigore, comunque svolgono importanti funzioni (Merton [1949]1970: 173 e passim; Aubert 1950; 1952; 1969; Thurman 1962; Murray 1964; Bettini 1983; Cotterrell 1992: 102-6). Alla luce delle menzionate riflessioni non può sfuggire come la funzione latente di una legge inefficace perché inapplicata ma formalmente in vigore, dunque l'effetto concreto da essa prodotto, consista in una riduzione del tasso di conflittualità che investe gli attori sociali caratterizzati da diseguale distribuzione di quote potere: i primi risultando soddisfatti dell'esistenza simbolica della legge, i secondi della sua pratica disapplicazione. Si tratta di un aspetto della dinamica del diritto vertente sul tema dell'efficacia giuridica sotto il profilo degli effetti prodotti su cui incidono variabili esterne al sistema giuridico, tipiche di altri sistemi di azione, come quello politico ed economico. Variabili in grado di suscitare ulteriori interrogativi dal punto di vista dell'efficacia giuridica (Febbrajo 2009: 110): rimane senza risposta il quesito se le norme formalmente in vigore ma del tutto inapplicate debbano o meno considerarsi efficaci.

\section{Conclusioni: per una definizione dell'efficacia giuridica}

Le riflessioni svolte sul tema dell'efficacia del diritto, costituendo il nucleo centrale della sociologiagiuridica in grado di distinguerla da altri discorsi riguardanti il diritto, suggeriscono l'opportunità di descrivere e indagare in modo analitico le variabili che sono in grado di influenzarla ricorrendo alla ricerca teorico-empirica (Treves 1977; 1987-88). Un compito che non appare sempre agevole poiché sulle variabili, sugli indici utilizzati per definirle e stabilirne l'incidenza, spesso pesano le intenzioni di coloro che operano sul materiale normativo, la cultura dei destinatari delle norme, le percezioni, le motivazioni comportamentali, elementi non sempre quantificabili in grandezze matematiche. Si tratta inoltre di variabili che non possono essere compiutamente comprese e spiegate se non si riflette sul fatto che il sistema giuridico, le norme che lo compongono, dipendono in larga misura da altri sistemi d'azione ad esso esterni ma mai estranei (Ferrari 
1997: 233). Spesso ci si è domandati se il diritto sia, rispetto alla "società", o rispetto ad altri sistemi sociali, una variabile dipendente o indipendente" (Treves 1966; 1968; 1975; 1976; 1977; 1982; 1987-88: 169-79), senza considerare che tale distinzione non può essere assunta in termini assoluti poiché se è vero che il diritto è influenzato da altri sistemi, vero è che a sua volta contribuisce ad influenzarli. Sotto questo profilo se non vi sono dubbi circa il fatto che il sistema giuridico dipenda in larga misura dal sistema culturale, burocratico, politico-istituzionale, sociale, militare, ecc., allora, e a maggior ragione, non vi possono essere incertezze, segnatamente si sottolinea, circa il fatto che il sistema giuridico dipende in ultima istanza dai sistemi economico e politico: rappresentando queste ultime altrettante variabili che, sommate a e confondendosi con quelle sopra descritte, appaiono in grado di influenzare l'efficacia giuridica. Sotto questo profilo occorre sottolineare come il giudizio di efficacia o inefficacia sul diritto verta innanzitutto e soprattutto, per ciò che concerne il quadro analitico dei fattori che appaiono maggiormente in grado di influenzarla, sulle variabili esterne al sistema giuridico, cioè riferibili a sistemi d'azione umana diversi dal diritto, tra cui, primariamente si annoverano il sistema politico ed economico. Ogni quesito riguardante l'efficacia del diritto se evidenzia il carattere strettamente politico ed economico che la connota, allora sottolinea, da una parte, la dipendenza di tale nozione dalla più ampia macro-variabile dei rapporti di potere, quest'ultimo inteso sia nelle sue più svariate forme (Ghezzi 2018: 34), materiali e simboliche, sia nell'accezione più anodina che lo rappresenta come facoltà di scelta tra alternative d'azione contrastanti (Lasswell [1948]1975; Russel [1938]1953; Oppenheim [1961]1964: 102-4; Lasswell \& Kaplan [1950]1969; Ferrari 1997: 143); dall'altra, la stretta colleganza che tale concetto ha, sotto il profilo degli effetti prodotti, con le funzioni di orientamento sociale, di trattamento dei conflitti dichiarati e di legittimazione del potere, ovvero e in termini riassuntivi con la funzione allocativa del diritto (Ferrari 1997: 252; Ghezzi 2018: 37): intendendosi con questa espressione, nella sua più ampia accezione, l'accesso a qualsiasi bene o il soddisfacimento di qualsiasi interesse in un mondo caratterizzato da scarsità e da conflitti (Ferrari 1997: 253). Sotto questo profilo il diritto dipende dal ed è orientato al conflitto sociale per l'acquisizione di risorse materiali, simboliche e posizionali, caratterizzate da scarsità, reale o presunta (Hirsch [1978]1981): il diritto, ancora oggi affermano in un'unica voce Vincenzo Tomeo e Bruno Bilotta, suo allievo diretto, è la “struttura del conflitto" (Tomeo 1981; Bilotta 1999; 2008a; 2008b; 2013; 2017), di quel conflitto che emerge dall'intreccio di molteplici processi d'azione, espressione dell'operare di innumerevoli variabili, dipendenti o 
indipendenti, riguardanti l'ordine sociale, culturale, militare, burocratico, istituzionale, politico ed economico, ecc. I caratteri assunti dal conflitto sociale, la struttura collettiva o individualistica, le relazioni fra le parti in conflitto, la distribuzione del potere nei e tra i gruppi di conflitto, sono solo alcuni degli elementi in grado di incidere sulle attività normative di tipo giuridico usualmente orientate a tenere sotto controllo gli antagonisti, a tracciare il limite fra la propria sfera d'azione e quella altrui, a produrre effetti sia in quella individuale, che in quella collettiva (Crespi 1994; Ferrari 1987; 1997: 233-4; 2006: 67; Ghezzi 2018: 33).

In questa prospettiva il sistema giuridico, le singole norme che lo costituiscono non sono soltanto un mezzo, il terreno di confronto e scontro ove si svolgono i processi d'azione orientati all'acquisizione di risorse scarse, ma esprimono anche un fine, un modello ideale capace di orientare l'azione umana e di esserne a sua volta orientato: norme e azioni, mezzi e fini, su cui pesa una moltitudine di variabili dipendenti e indipendenti, che, alla luce degli interessi e scopi perseguiti dagli attori sociali attraverso di esse, sostanziano sotto il profilo degli effetti realizzati o da realizzare le funzioni svolte o che si intendono svolgere, ovvero e in una parola l'efficacia del diritto (Calamandrei 1950; Carnelutti 1953; Huizinga [1939]1975; Andrini 1991). In questi termini il giudizio di efficacia delle norme consiste proprio nella maggiore o minore corrispondenza fra il quadro ideale, antecedente e conseguente all'azione, e il quadro effettivo a questa riconducibile, dunque nella distanza che corre tra il diritto in azione e quello nei libri (Pound 1910: 12 ss.).

In questa prospettiva, valida per ogni tipo di rapporto giuridico, dal processo giudiziario, alla formazione di un contratto, sino ad arrivare all'emanazione di una legge, finanche di rango costituzionale, appare evidente che, come ha bene osservato Geiger, le azioni degli attori sociali in rapporto al diritto non possono essere spiegate e comprese utilizzando unicamente la logica dicotomica trasgressione e ottemperanza, comminatoria o meno di una sanzione, cui sembra ridursi ogni decisione giuridica, compresa quella riguardante il giudizio di efficacia sul diritto: lo spazio intermedio risulta essere decisamente più importante degli estremi teorici considerati, poiché sia l'uno che gli altri, come del resto accade per le regole e la struttura dell'ordinamento giuridico, sono oggetto di un continuo mutamento in funzione delle variabili maggiormente in grado di determinarlo (Van de Kerchove \& Ost, [1992]1995; Ost. \& Van de Kerchove 1997: 7 ss.; Merelau-Ponty 1964: 75 ss). 
Le riflessioni svolte ovviamente non intendono sostenere l'idea che l'interazione sociale in rapporto al diritto, il giudizio di efficacia sulle norme si caratterizzino per un'incertezza, una contingenza, un'insicurezza e un'incomunicabilità assolute, perché è esattamente vero il contrario: gli attori sociali hanno interesse a comprendere e a farsi comprendere, a garantire la stabilità, la certezza e la sicurezza dei rapporti umani, a rispettare e far rispettare il diritto, in definitiva ad esercitare il potere. Occorre tuttavia sottolineare come il rischio di incomprensione comunicativo-normativa e di incertezza comportamentale riguardante il diritto e il giudizio di efficacia sotto il profilo dell'ottemperanza-trasgressione di una norma, della relativa comminatoria della sanzione tenda ad acuirsi notevolmente allorché l'azione comunicativa verta su norme con una struttura deontica: volta ad esprimere prescrizioni rivolte a un soggetto da parte di un altro ovvero a produrre effetti nella sfera d'azione altrui tracciandone i rispettivi confini (Ferrari 1997: 168). Nella comunicazione prescrittiva indirizzata a influenzare e a qualificare l'azione altrui, a produrre effetti nell'altrui sfera d'azione, il soggetto che invia o riceve il messaggio normativo normalmente prospetta un significato il più possibile aderente agli interessi e scopi perseguiti attraverso di esso, spesso diversi se non del tutto divergenti rispetto a quelli perseguiti dall'interlocutore antagonista o opposto (Di Lucia 1996: 38 ss.; Conte 2011: 58 ss). In questo senso come ha chiaramente rilevato Geiger il conflitto vertente sul diritto, la contesa tra le parti parlamentari per una legge, la disputa tra quelle contrattuali per un contratto, lo scontro tra le parti processuali, la discussione dottrinale e quella giurisprudenziale, etc.., in relazione agli effetti che si intendono realizzare hanno sempre per oggetto dei significati normativi, trattandosi di conflitto semiotico vertente su segni sia pur riferito a fatti concreti e fattispecie astratte (Ferrari 1997: 168). In questi termini e sulla scorta delle riflessioni svolte da Geiger sul tema è possibile definire l'efficacia del diritto come una relazione sociale di potere, soprattutto comunicativa, che si risolve in una scelta e contrapposizione di segni o simboli da parte dei soggetti agenti, nel significato più aderente possibile agli interessi e scopi perseguiti attraverso di esse, agli effetti prodotti o che si intendono produrre mediante il loro utilizzo, al fine di garantire l'accesso a qualsiasi bene o il soddisfacimento di ogni interesse in un mondo caratterizzato da scarsità e da conflitti.

\section{Riferimenti bibliografici}

Andrini, Simona, 1991. Huizinga et le droit: le procés et le jeu en Italie. Droit et Société, 17-18: 25-37.

Aubert, Vilhelm, 1950. Priskontroll og rasjonering. En rettsociologisk forstudie. Oslo: Akademisk Forlag. 
—, Torstein Eckhoff \& Knut Sveri, 1952. En lov i sokelyset. Sosialpsykologisk undersokelse av den norske huisjelplov. Oslo: Akademisk Forlag.

—, 1969. Introduction. In Vilhelm Aubert (ed.), Sociology of Law. Selected Readings. Harmondsworth: Penguin.

Bardach, Eugene, 1977. The Implementation Game. What happens after a Bill Becomes Law. Cambridge (Mass): MIT Press.

Berger, Peter Ludwig \& Thomas Luckmann, [1966]1969. The Social Construction of reality. Traduzione italiana La realtà come costruzione sociale. A cura di Marta Sofri Innocenti \& Alessandra Sofri Peretti, Bologna: Il Mulino.

Bettini, Romano, 1983. Il circolo vizioso legislativo. Efficacia del diritto ed efficienza degli apparati pubblici in Italia. Milano: Angeli.

—, 1988. Analisi funzionale ed efficacia del diritto. Rivista internazionale di filosofia del diritto, 1: 3-15.

—, 1990. Legislazione e politiche i Italia. Razionalità, efficacia, modernizzazione imperfetta. Milano: Angeli.

Bilotta, Bruno M. (a cura di), 1999. La giustizia alternativa. Torino: Giappichelli.

—, (a cura di) 2008a. Forme di giustizia tra mutamento e conflitto sociale. Milano: Giuffrè.

—, (a cura di) 2008b. Conflitti e istanze di giustizia nelle società contemporanee. Milano: Giuffrè.

—, 2013. Ripensare al diritto come struttura del conflitto. Premessa. In Vincenzo Tomeo, Il diritto come struttura del conflitto. Una analisi sociologica. Soveria Mannelli: Rubettino.

—, 2017. Per una sociologia dei conflitti. Una proposta. In Bruno M. Bilotta (a cura di) Elementi di sociologia dei conflitti. Padova: CEDAM.

Blankenburg, Erhard, 1986. La recherche de l'efficacitè de la loi. Rèflexions sur l'ètude de la mise en oeuvre: le concept d'implèmentation. Droit et Sociètè, 2: 59-75.

—, 1988. Implémentation. In André-Jacques Arnaud (éd.) Dictionnaire encyclopédique de théorie et de sociologie du droit. Paris: Librairie Générale de Droit et de Jurispridence et Story Scientia. 
—, 1995. Mobilisierung des Rechts. Berlin-Heidelberg-New York: Springer.

Bosello, Furio, 1990. La certezza nei rapporti tributari. Sociologia del diritto. 17, 1-2: 25-56.

Calamandrei, Pietro, 1950. Il processo come giuoco. In Aa. Vv., Studi in onore di Francesco Carnelutti. Padova: Cedam.

Candian, Albina, Antonio Gambaro \& Barbara Pozzo, 1992. Property-Propriété-Eigentum. Corso di diritto privato comparato. Padova: Cedam.

Carnelutti, Francesco, 1953. Giuoco e processo. In Aa. Vv., Studi in onore di Vincenzo Arangio Ruiz, Napoli: Jovene.

Conte, Amedeo Giovanni, 1962. Saggio sulla completezza degli ordinamenti giuridici. Torino: Giappichelli.

_, 1986. Fenomeni di fenomeni. Rivista internazionale di filosofia del diritto, 63: 29-57.

—, 1995. Filosofia del linguaggio normativo. Torino: Giappichelli.

—, 2011. Sociologia filosofica del diritto. Torino: Giappichelli.

Cotterrell, Roger, 1992. Sociology of Law. An Introduction. London: Butterworths.

Crespi, Franco, 1994. Le vie della sociologia. Bologna: Il Mulino.

D’Alessandro, Lucio, 2018. Diritto e società. Per un immaginario della cultura giuridica. Napoli: Guida Editori.

Damiani di Vergada Franzetti, Enrico, 2018. Definire la sociologia del diritto: riflessioni e spunti. In Marco A. Quiroz Vitale \& Enrico Damiani di Vergada Franzetti (a cura di), Dossier. Renato Treves tra filosofia e sociologia, in Europa e America Latina. Sociologia del diritto (numero monografico), 45, 2: 154-164.

Di Lucia, Paolo, 1996. Agire secondo una norma, agire per una norma, agire in funzione di una norma. In Paolo Comanducci \& Riccardo Guastini (a cura di), Struttura e dinamica dei sistemi giuridici. Torino: Giappichelli.

Febbrajo, Alberto, 1979. Materiali sulla sociologia del diritto di Theodor Geiger. Pavia: Edizioni universitarie Gjes. 
—, 1984. Storia e sociologia del diritto. Milano: Giuffrè.

—, [2009]2013, Sociologia del diritto. Concetti e Problemi. Bologna: Il Mulino.

—, 2018. Theodor Geiger e il modello di una cultura giuridica "riflessiva". In Theodor Geiger, Vorstudien zu einer Soziologie des Rechts. Traduzione italiana Studi preliminari di sociologia del diritto. A cura di Morris Ghezzi, Nicoletta Bersier Ladavac e Michele Marzulli. Milano: Mimesis.

Ferrari, Vincenzo, 1987. Funzioni del diritto. Roma-Bari: Editori Laterza.

—, 1997, Lineamenti di sociologia del diritto. Milano: Editori Laterza.

Fittipaldi, Edoardo, 2012. Everyday Legal Ontology: A Psichological and Linguistic Investigation within the Framework of Leon Petrażycki’s Theory of Law. Milano: LED.

-, 2016. Leon Petrażycki. In Enrico Pattaro \& Corrado Roversi, C., (a cura di), Legal Philosophy in the Twentieth Century: The Civil Law World. Berlin: Spinger.

Friedman, Lawrence M., [1975]1978. The Legal System. A Social Science Perspective. Traduzione italiana Il sistema giuridico nella prospettiva delle scienze sociali. A cura di Giovanni Tarello. Bologna: Il Mulino.

Galtung, Johan, 1960. Expectations and Interaction Process. Inquiry, 2: 213-234.

Geiger, Theodor, [1947]1964a. Vorstudien zu einer Soziologie des Rechts. Neuwied: Luchterhand.

-, [1947]1964b. Vorstudien zu einer Soziologie des Rechts. Copenhagen: Acta Jutlandica

-, [1947]2018. Vorstudien zu einer Soziologie des Rechts. Traduzione italiana Studi preliminari di sociologia del diritto. A cura di Morris L. Ghezzi, Nicoletta Bersier Ladavac \& Michele Marzulli. Milano: Mimesis.

Ghezzi, Morris L., 1995. Diversità e pluralismo. La sociologia del diritto penale nello studio di devianza e criminalità. Milano: Cortina.

-, 2017. Ciò che resta. La rivoluzione del diritto come estetica. Milano: Mimesis. 
-, 2018. Theodor Geiger e la sociologia del diritto come antimetafisica sociale. In Theodor J. Geiger, Studi preliminari di sociologia del diritto. A cura di Morris L. Ghezzi, Nicoletta Bersier Ladavac \& Michele Marzulli. Milano: Mimesis.

Hart, Herbert L. A., [1961]1965. The Concept of Law. Traduzione italiana Il concetto di diritto. Torino: Einaudi.

Hirsch, Fred, [1978]1981. Social Limits to Growth. Traduzione italiana Limiti sociali allo sviluppo. Milano: Bompiani.

Huizinga, John, [1939]1975. Homo Ludens. Traduzione italiana Homo Ludens. Torino: Einaudi.

Jackson, Bernard S., 1985. Semiotics and Legal Theory. London-Boston-Melbourne-Henley: Routledge \& Kegan Paul.

Jori, Mario \& Anna Pintore, 1995. Manuale di teoria generale del diritto. Torino: Giappichelli.

Kagan, Robert A., Eugene Bardach, 1982. Going by the Book. The Problem of Regulatory Unreasonableness. Philadelphia: Temple University Press.

Kalinowskj, Georges, 1993. Sémiotique juridique. In André-Jacques Arnaud (éd.), Dictionnaire encyclopédique de Théorie et de sociologie du droit. Paris: Libraire Gènèrale de Droit et de Jurisprudence.

Kelsen, Hans, 1967. Lineamenti di dottrina pura del diritto. Torino: Einaudi.

-, [1979]1985. Allgemeine Theorie der Normen. Traduzione italiana Teoria generale delle norme. A cura di Mario G. Losano. Torino: Einaudi.

Kerchove, Michel van de, François Ost, [1992]1995. Le Droit ou les paradoxes du jeu. Traduzione italiana Il diritto ovvero i paradossi del gioco. Milano: Giuffrè.

Lasswell, Harold Dwight, [1948]1975. Power and Personality. Traduzione italiana Potere e personalità. Torino: Utet.

-, \& Abraham Kaplan, [1950]1969. Traduzione italiana Potere e società. Milano: Etas Kompass.

Mayntz, Renate (Hrsg.), 1980. Implementation politischer Programme. Konigsstein: Athenaum. 
Marx, Karl, [1842]1975. Debatten über das Holzdiebstahlsgesetz. Traduzione italiana Dibattiti sulla legge contro i furti di legna. In Karl Marx, Scritti politici giovanili. A cura di Luigi Firpo. Torino: Einaudi.

Merleau-Ponty, Maurice, 1964. Interrogation et dialectique. In Maurice Merleau-Ponty, Le visible et l'invisible. Paris: Gallimard.

Merton, Robert K., [1949]1970. Social Theory and Social Structure. Traduzione italiana Teoria e struttura sociale. Bologna: Il Mulino.

Morris, Charles, 1938. Foundations of the Theory of Signs. Chicago: The University of Chicago Press.

Murray, Edelman, 1964. The Symbolic Uses of politics. Urbana: University of Illinois Press.

Nelken, David, 1993. The Truth about Law's Truth. In Alberto Febbraio, David Nelken (eds.), European Yearbook in the Sociology of Law.

Oppenheim Felix, [1961]1964. Dimensions of Freedom. Traduzione italiana Dimensioni della libertà. Milano: Feltrinelli.

Ortega Y Gasset, Josè, 1946-1947. El tema de nuestro tempo. In Josè Ortega y Gasset, Obras completas, Madrid: Revista Occidente.

Ost, François, Michel de Kerchove, 1997. Pensare la complessità del diritto: per una teoria dialettica. Sociologia del diritto, 24, 1: 5-26.

Paliero, Carlo Enrico, 1990. Il principio di effettività del diritto penale. Rivista italiana di diritto e procedura penale, 33: 430-544.

Petrażycki, Leon, 1909-1910. Teorija prava i gosudarstva v svjazi s teoriej nravstvennosti. Sankt-Peterburg: Ekateringofskoe Pec'atnoe Delo.

Podgòrecki, Adam, 1991. A Sociological Theory of Law. Milano: Giuffrè.

-, Jon Alexander \& Rob Shields, (eds.), 1996. Social Engineering. Ottawa: Carleton University Press.

Pound, Nathan Roscoe, 1910. Law in Books and Law in Action. American Law Reviev, 44: 12-46. 
Russel, Bertrand, [1938]1953. Power. A New Social Analysis. Traduzione italiana Il Potere. Una nuova analisi sociale. Milano: F.lli Bocca.

Scarpelli, Uberto, [1959]1985. Contributo alla semantica del linguaggio normativo. Milano: Giuffrè.

-, 1969. Semantica giuridica. In Novissimo Digesto italiano, vol. 16. Torino: Utet.

Sgubbi, Filippo, 1990. Il reato come rischio sociale. Ricerche sulle scelte di allocazione dell'illegalità penale. Bologna: Il Mulino.

Tarello, Giovanni, 1975. Diritto, enunciati, usi. Studi di teoria e metateoria del diritto. Bologna: Il Mulino.

-, 1980. L'interpretazione della legge. In Antonio Cicu \& Francesco Messineo (a cura di) Trattato di diritto civile e commerciale, v. 5. Milano: Giuffrè.

-, 1990. L'interpretazione della legge. Milano: Giuffrè.

Tomeo, Vincenzo, 1981. Il diritto come struttura del conflitto. Un'analisi sociologica. Soveria Mannelli: Rubbettino.

Treves, Renato (a cura di), 1966. La sociologia del diritto. Problemi e ricerche. Milano: Edizioni di Comunità.

-, (a cura di), 1968. Nuovi sviluppi della sociologia del diritto. Milano: Edizioni di Comunità.

-, 1975. Giustizia e giudici nella società italiana. Problemi e ricerche di sociologia del diritto. Bari: Laterza.

-, (a cura di), 1976. L'insegnamento sociologico del diritto. Milano: Edizioni di Comunità.

-, 1977. Introduzione alla sociologia del diritto. Torino: Einaudi.

-, 1982. La sociologia del diritto in Italia oggi. Napoli: Guida Editori.

-, 1987-1988. La sociologia del diritto. Origini, ricerche, problemi. Torino: Einaudi.

Thurman, Arnold, 1962. The Symbols of Government. New York: Brace and World. 